\title{
Accurate Fault Location for Double-Circuit Lines on the Same Tower with Asymmetrical Parameters
}

\author{
ZHU Yiyang ${ }^{1, a^{*}}$, DU Hongji, ${ }^{1, b}$ \\ ${ }^{1}$ Nanjing University of Technology and Engineering, Nanjing 210094, China

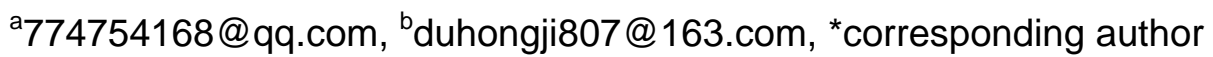

\begin{abstract}
Keywords: Asymmetrical parameter; double-circuit lines on the same tower; zero mutual inductance; decouple; fault location
\end{abstract}

\begin{abstract}
Traditional six-sequence components can only be applied to the double-circuit lines with symmetrical parameters. In reality, there may appear partially coupled lines with asymmetrical parameter because of the difference in construction. A new decoupling method is adopted to solve this problem. Three phases are first decoupled to positive , negative and zero sequence component , but there are mutual inductance between zero sequence component. Then the zero sequence is divided into the same direction component and the reverse component . The method solves the problem of zero sequence mutual inductance of double circuit lines on the same tower with asymmetric parameters. The current and voltage of both ends of the transmission line can be decoupled into the new six sequence component by new six-sequence component method. The fault location formula can be obtained based on that the sequence voltage of fault point calculated by both ends are equivalent. PSCAD simulation results show that the method can accurately measure the distance of fault and is not affected by fault type, fault location , fault resistance and other factors.
\end{abstract}

\section{Introduction}

Now the land resource is in short supply, the cost of setting up the transmission line is getting higher and higher, and the transmission capacity is increasing day by day. The double circuit lines on the same tower can improve the transmission capacity of the transmission corridor, so it has been widely used. When a fault occurs in power system, accurate fault location can quickly locate the fault position and the fault can be removed in time. It can save a lot of manpower and material resources, and reduce the economic losses due to power failure. Fault location of double circuit lines is difficult because of more fault types and interaction between lines. Most of ranging methods have been proposed for double circuit lines on the same tower are based on the condition of symmetric parameter. If the line parameters are asymmetrical, the coupling relationships will be more complex, and more fault types will exist. It is necessary to study a measurement method of double circuit transmission lines with asymmetric parameters.

At present, the fault location is mainly divided into single-ended method[1,2] and double-ended method[3,4]. Single-ended method just uses data of one end of the line, the realization is simple, but it is influenced by the transition resistance. Double-ended method is not affected by fault types and system impedance, the distance measurement is more accurate, but data synchronization of two ends is needed. According to characteristics of double circuit lines on the same tower based on distributed parameter model using asymmetrical parameter, the paper[5] proposes a new fault locating algorithm for double circuit lines on the same tower using asynchronous data of two terminals. The paper [6] proposes a new algorithm to solve simple faults of the double circuit lines on the same tower, and the symmetry of the power system parameters is not required.

Because the coupling of the double circuit lines is complicated, the six sequence[7,8] component method is used to decouple. The paper[9] proposes a decoupling method based on six sequence components, then uses asynchronous data of two terminals for fault location. But the six sequence component method is generally applied to the double circuit lines with symmetrical parameters. It is no longer suitable for the double circuit lines with asymmetrical parameters. The paper[10] proposes 
a new sequence component method applied to double circuit lines on the same tower with asymmetrical parameters. In order to fully decouple the impedance matrix with asymmetrical parameters, the zero sequence four component method is proposed in paper [11].

The current and voltage of both ends of the transmission line can be decoupled into the new six sequence components by new decoupling method in this paper. The fault location formula can be established based on that the sequence voltage of fault point calculated by both ends are equivalent . Accurate fault distance can be obtained with the formula. The effectiveness of the proposed method is verified by PSCAD simulation.

\section{Characteristics of Double Circuit Lines with Asymmetrical Parameters}

The model diagram of the double circuit transmission line is shown in Fig.1.The two circuit lines are coupled with each other. Where $E_{m} 、 E_{n}$ is respectively power supply voltage of two ends of transmission line.is the impedance of line

I .is the impedance of line II.

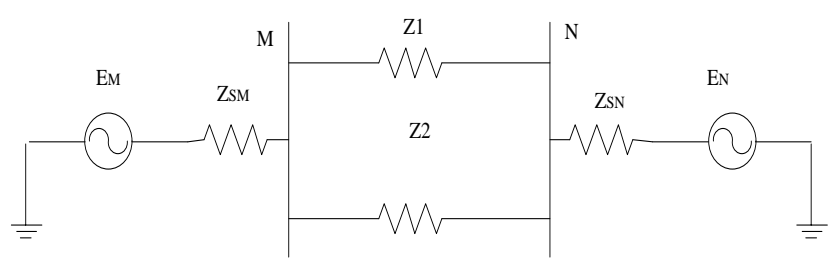

Fig. 1 System structure diagram

Fig. 2 shows the parameters of MN part of the line. The parameters of the two circuit lines are different, but the parameters of the circuit are symmetrical. Where $Z_{l 1}$ is the self inductance of line I, $Z_{m 1}$ is the mutual inductance of line $\mathrm{I}, Z_{12}$ is the self inductance of line II, $Z_{m 2}$ is the mutual inductance of line II, $Z_{p}$ is the self inductance of two lines.

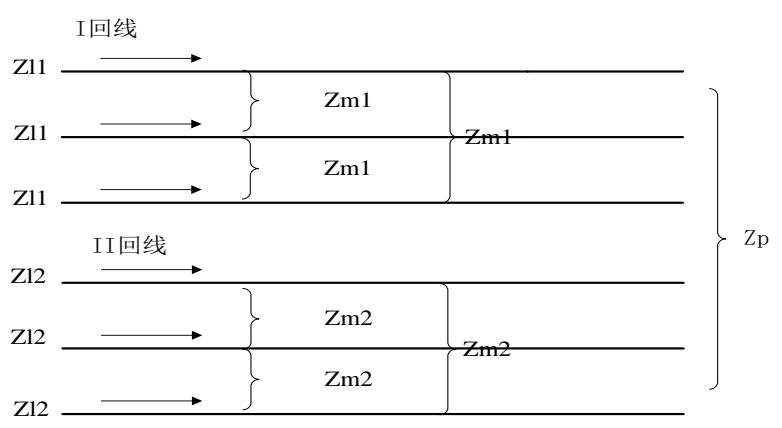

Fig. 2 Line parameter

From Fig.2, the relationship between voltage and current of the double circuit line is:

$$
\left[\begin{array}{c}
\Delta U_{\mathrm{IA}} \\
\Delta U_{\mathrm{IB}} \\
\Delta U_{\mathrm{IC}} \\
\Delta U_{\mathrm{IIA}} \\
\Delta U_{\mathrm{IIB}} \\
\Delta U_{\mathrm{IIC}}
\end{array}\right]=\left[\begin{array}{cccccc}
Z_{l 1} & Z_{m 1} & Z_{m 1} & Z_{p} & Z_{p} & Z_{p} \\
Z_{m 1} & Z_{l 1} & Z_{m 1} & Z_{p} & Z_{p} & Z_{p} \\
Z_{m 1} & Z_{m 1} & Z_{l 1} & Z_{p} & Z_{p} & Z_{p} \\
Z_{p} & Z_{p} & Z_{p} & Z_{l 2} & Z_{m 2} & Z_{m 2} \\
Z_{p} & Z_{p} & Z_{p} & Z_{m 2} & Z_{l 2} & Z_{m 2} \\
Z_{p} & Z_{p} & Z_{p} & Z_{m 2} & Z_{m 2} & Z_{l 2}
\end{array}\right]\left[\begin{array}{l}
I_{\mathrm{IA}} \\
I_{\mathrm{IB}} \\
I_{\mathrm{IC}} \\
I_{\mathrm{IIA}} \\
I_{\mathrm{IIB}} \\
I_{\mathrm{IIC}}
\end{array}\right]
$$

Where $\Delta U_{\text {IA }} 、 \Delta U_{\text {IB }} 、 \Delta U_{\text {IC }} 、 \Delta U_{\text {IIA }} 、 \Delta U_{\text {IIB }} 、 \Delta U_{\text {IIC }}$ is voltage drop of the line, $\Delta U_{\text {IA }} 、 \Delta U_{\text {IB }}$ 、 $\Delta U_{\text {IC }} 、 \Delta U_{\text {IIA }} 、 \Delta U_{\text {IIB }} 、 \Delta U_{\text {IIC }}$ is circuit of the line.

The impedance matrix is marked as $Z$, above formula can be simplified as $\Delta U_{A B C}=Z{ }^{*} I_{A B C}$.

Where $\Delta U_{A B C}$ is voltage drop of the line, $I_{A B C}$ is circuit of the line. 


\section{The Decoupling Method of Line}

Traditional Six-Sequence Components Method. The characteristics of double circuit transmission lines on the same tower are two lines coupling with each other. That means each phase owns self inductance and mutual inductance between each other. There are also mutual inductance between lines. The six sequence component method is applied to the double circuit lines with symmetrical parameters. The self inductance and mutual inductance of the line are equivalent. The essence of the six sequence component method is to eliminate the mutual inductance between lines. First realize the decoupling between the lines, the voltage and current of the double lines is divided into the same direction vector $\mathrm{T}$ and reverse direction vector $\mathrm{F}$.

Then realize the decoupling between the phases. The same direction vector and reverse direction vector can be divided into the same direction positive sequence, the same direction negative sequence, the same direction zero sequence, reverse direction positive sequence, reverse direction negative sequence and reverse direction zero sequence. These six components are independent of each other.

Decoupling Method Of Double Circuit Lines With Asymmetrical Parameters. Due to the asymmetry of the double circuit lines parameters, lines can not be decoupled first as six sequence component method. The decoupling between the phases can be realized first with the method proposed by paper[9].The double circuit lines are divided into positive sequence, negative sequence and zero sequence component with matrix $Q$.

$$
Q=\left[\begin{array}{cccccc}
1 & 1 & 1 & & & \\
1 & a^{2} & a & & & \\
1 & a & a^{2} & & & \\
& & & 1 & 1 & 1 \\
& & & 1 & a^{2} & a \\
& & & 1 & a & a^{2}
\end{array}\right]
$$

The formula (1) can be expanded as follows:

$$
\left[\begin{array}{c}
\Delta U_{\mathrm{I} 0} \\
\Delta U_{\mathrm{I} 1} \\
\Delta U_{\mathrm{I} 2} \\
\Delta U_{\mathrm{II}} \\
\Delta U_{\mathrm{II} 1} \\
\Delta U_{\mathrm{II} 2}
\end{array}\right]=\left[\begin{array}{llllll}
Z_{\mathrm{I} 0} & & & 3 Z_{p} & & \\
& Z_{\mathrm{I} 1} & & & & \\
& & Z_{\mathrm{I} 2} & & & \\
3 Z_{p} & & & Z_{\mathrm{II} 0} & & \\
& & & & Z_{\mathrm{II} 1} & \\
& & & & & Z_{\mathrm{II} 2}
\end{array}\right]\left[\begin{array}{c}
I_{\mathrm{I} 0} \\
I_{\mathrm{I} 1} \\
I_{\mathrm{I} 2} \\
I_{\mathrm{II}} \\
I_{\mathrm{II} 1} \\
I_{\mathrm{II} 2}
\end{array}\right]
$$

Where zero sequence impedance of $\mathrm{I}$ is $Z_{\mathrm{I} 0}=Z_{l 1}+2 Z_{m 1}$, zero sequence impedance of II is $Z_{\mathrm{II} 0}=Z_{l 2}+2 Z_{m 2}$, positive and negative sequence impedance of $\mathrm{I}$ is $Z_{\mathrm{I} 1}=Z_{\mathrm{I} 2}=Z_{l 1}-Z_{m 1}$, positive and negative sequence impedance of II is $Z_{\mathrm{II} 1}=Z_{\mathrm{II} 2}=Z_{l 2}-Z_{m 2}$.

Formula(2)shows positive and negative sequence components are independent of each other, but there are mutual inductance between zero sequence components. Zero sequence components need to be decoupled. The impedance matrix needs diagonalization processing. And that processing, the corresponding characteristic value is the impedance value on the diagonal. Corresponding feature vectors can be composed of a decoupled matrix $M$. The zero sequence component is decoupled by $M$.<smiles>[M]C1CCCC1</smiles> 


$$
\begin{gathered}
k_{1}=\frac{Z_{\mathrm{II} 0}-Z_{\mathrm{I} 0}+\sqrt{\left(Z_{\mathrm{II} 0}-Z_{\mathrm{I} 0}\right)^{2}+36 * Z_{p}^{2}}}{6 Z_{p}} \\
k_{2}=\frac{Z_{\mathrm{II} 0}-Z_{\mathrm{I} 0}-\sqrt{\left(Z_{\mathrm{II} 0}-Z_{\mathrm{I} 0}\right)^{2}+36 * Z_{p}^{2}}}{6 Z_{p}}
\end{gathered}
$$

The formula (2)can be expanded as follows:

$$
\left[\begin{array}{c}
\Delta U_{01} \\
\Delta U_{\mathrm{II}} \\
\Delta U_{\mathrm{I} 2} \\
\Delta U_{02} \\
\Delta U_{\mathrm{II} 1} \\
\Delta U_{\mathrm{II} 2}
\end{array}\right]=\left[\begin{array}{llllll}
Z_{01} & & & & & \\
& Z_{\mathrm{I} 1} & & & & \\
& & Z_{\mathrm{I} 2} & & & \\
& & & Z_{02} & & \\
& & & & Z_{\mathrm{II} 1} & \\
& & & & & Z_{\mathrm{II} 2}
\end{array}\right]\left[\begin{array}{c}
I_{01} \\
I_{\mathrm{I} 1} \\
I_{\mathrm{I} 2} \\
I_{02} \\
I_{\mathrm{II} 1} \\
I_{\mathrm{II} 2}
\end{array}\right]
$$

Where $\Delta U_{01} 、 \Delta U_{02}$ is the first and second zero sequence component of voltage after decoupling, $I_{01} 、 I_{02}$ is the first and second zero sequence component of current after decoupling, $Z_{01} 、 Z_{02}$ is the first and second zero sequence component of impedance after decoupling.

$$
\begin{aligned}
& Z_{01}=\left(Z_{\mathrm{II} 0}+Z_{\mathrm{I} 0}+\sqrt{\left(Z_{\mathrm{II} 0}-Z_{\mathrm{I} 0}\right)^{2}+36 * Z_{p}^{2}}\right) / 2 \\
& Z_{02}=\left(Z_{\mathrm{II} 0}+Z_{\mathrm{I} 0}-\sqrt{\left(Z_{\mathrm{II} 0}-Z_{\mathrm{I} 0}\right)^{2}+36 * Z_{p}^{2}}\right) / 2
\end{aligned}
$$

The total decoupling matrix is:

$$
N=\mathrm{QM}=\left[\begin{array}{cccccc}
1 & 1 & 1 & 1 & & \\
1 & a^{2} & a & 1 & & \\
1 & a & a^{2} & 1 & & \\
k_{1} & & & k_{2} & 1 & 1 \\
k_{1} & & & k_{2} & a^{2} & a \\
k_{1} & & & k_{2} & a & a^{2}
\end{array}\right]
$$

01, 02, I 1, I $2, \mathbb{I} 1, \mathbb{I} 2$ the six independent sequence components can be got after decoupling by $\mathrm{N}$.

Fault Location Principle Of Double Circuit Lines On The Same Tower.Fig. 3 shows fault location principle of double circuit lines on the same tower, considering the influence of distributed capacitance. Where $Z_{i}$ is sequence impedance of each line, $Y_{i}$ is sequence admittance of each line, $L$ is total length of line, $\mathrm{D}$ the distance from the $\mathrm{M}$ side bus to the fault point, $Z_{M S i}$ and $Z_{N S i}$ are system impedance, $R_{f}$ is transition resistance.

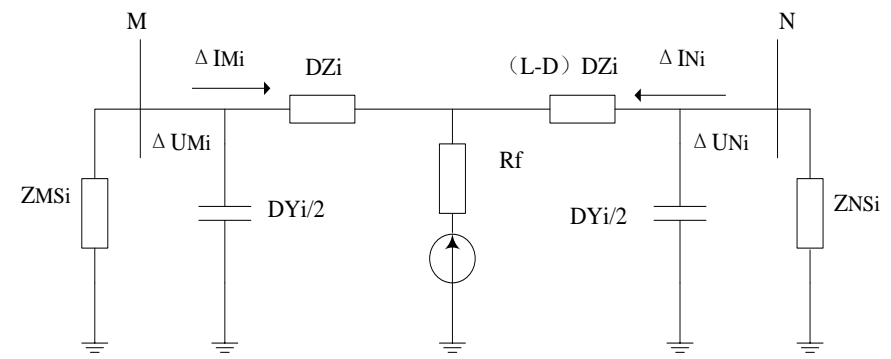

Fig. 3 Mutation sequence network of parallel lines

Voltage and current values are recorded before and after the failure of both sides of the bus, the fault value is equal to the subtraction of which. Six independent sequence components of voltage and current can be got after decoupling by matrix $\mathrm{N}$. The fault location formula can be obtained based on that the sequence voltage of fault point calculated by both ends are equivalent.

$$
\left|\Delta U_{M i}-\left(\Delta I_{M i}-\Delta U_{M i} \frac{D Y_{i}}{2}\right) D Z_{i}\right|=\left|\Delta U_{N i}-\left(\Delta I_{N i}-\Delta U_{N i} \frac{D Y_{i}}{2}\right)(L-D) Z_{i}\right| .
$$


Formula (8) shows that if the fault voltage and current of both ends of the line is fixed, $\left|U_{F M i}\right|$ decreases with the increase of $D$ and $\left|U_{F N i}\right|$ increases with the increase of $D$. So there will only be the only solution and there will be no pseudo root. In practical solution, a global one-dimensional search of $D$ can be carried out in the range of $0 \sim L$.Search step is $\Delta d . D$ is the correct fault distance when the difference value on both sides of formula (8) is minimal.

\section{The Simulation Verification}

The simulation system diagram is shown in Figure 1 . The length of double circuit line is $100 \mathrm{~km}$. The

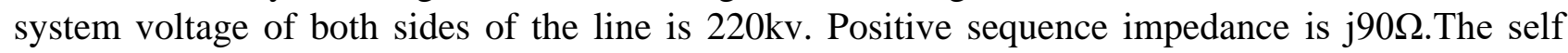
inductance of line $\mathrm{I}$ is $Z_{l 1}=(25.76+\mathrm{j} 132.55) \Omega$, the mutual inductance of line $\mathrm{I}$ is $Z_{m 1}=(18.25+\mathrm{j} 47.86) \Omega$, the self inductance of line II is $Z_{l 2}=(22.56+\mathrm{j} 112.37) \Omega$, the mutual inductance of line II is $Z_{m 2}=(15.34+\mathrm{j} 40.33) \Omega$, the self inductance of two lines is $Z_{p}=(25.68+\mathrm{j} 142.93) \Omega$.

Fault Location of Different Fault Type. The length of the line is $100 \mathrm{~km}$. Different types of faults occurred in $30 \mathrm{~km}$ and $50 \mathrm{~km}$ respectively. Fault location are carried out by using the negative sequence component of line I. The results of simulation and calculation are shown in table 1. From table 1,it can be known that the error of fault location based on the fault location method proposed in this paper is within $1 \%$.

Tab.1 Fault location of different fault type

\begin{tabular}{cccc}
\hline Fault Type & $\begin{array}{c}\text { Actual Fault } \\
\text { Distance[km] }\end{array}$ & $\begin{array}{c}\text { Fault } \\
\text { Location[km] }\end{array}$ & Error[\%] \\
\hline \multirow{2}{*}{ I AG } & 30 & 30.13 & 0.13 \\
& 50 & 49.86 & 0.14 \\
I BC & 30 & 30.25 & 0.25 \\
& 50 & 50.17 & 0.17 \\
I BCG & 30 & 30.16 & 0.16 \\
\multirow{2}{*}{ I B II C } & 50 & 50.23 & 0.23 \\
& 30 & 29.95 & 0.05 \\
I B II CG & 50 & 50.06 & 0.06 \\
& 30 & 30.18 & 0.18 \\
I A II BCG & 30 & 50.27 & 0.27 \\
& 50 & 29.89 & 0.11 \\
I AB II BCG & 30 & 49.79 & 0.21 \\
& 50 & 30.12 & 0.12 \\
\hline
\end{tabular}

Notes: I AG means A phase of line I to ground short circuit ; I BC means $A$ phase and $B$ phase of line I short circuit; I BCG means $B$ phase and $C$ phase of line I to ground short circuit; I B II CG means $B$ phase of line I and $C$ phase of line II to ground short circuit; I A II BCG means $A$ phase of line I, $B$ phase and $C$ phase of line II to ground short circuit.

Fault Location of Different Transition Resistance. The length of the line is $100 \mathrm{~km}$. Different types of grounding faults occurred in $30 \mathrm{~km}$ with different transition resistance. Fault location are carried out by using the negative sequence component of line I. The results of simulation and calculation are shown in table 2 . From table 2, it can be known that the method proposed in this paper is not affected by the transition resistance.

Fault Location of Different Sequence Component .The length of the line is $100 \mathrm{~km}$. A variety of metal grounding fault occurs in $30 \mathrm{~km}$.Fault location are carried out by using different sequence components. The results of simulation and calculation are shown in table 3.From table 3, it can be known that the error of fault location with positive sequence is larger and the range accuracy of 
negative sequence component and zero sequence component is relatively higher. Positive sequence component is widespread. When the negative sequence component is used for fault location , if the single circuit line fails, it is required to determine which circuit the fault occurred in . Zero sequence component is zero in the event of symmetrical faults and non ground faults. So in the actual situation, the negative sequence component and the zero sequence component after decoupling can be used for ranging.

\begin{tabular}{ccc}
\multicolumn{3}{c}{ Tab.2 Fault location of different transition resistance } \\
\hline Fault Type & Fault Location[km] & Error[\%] \\
\hline \multirow{2}{*}{ I AG } & 30.17 & 0.17 \\
& 30.18 & 0.18 \\
& 30.22 & 0.22 \\
I BCG & 29.87 & 0.13 \\
& 30.19 & 0.19 \\
I B II CG & 30.14 & 0.14 \\
& 29.79 & 0.21 \\
& 29.86 & 0.14 \\
I A II BCG & 29.85 & 0.15 \\
& 30.11 & 0.11 \\
\hline
\end{tabular}

Tab.3 Fault location of different sequence component

\begin{tabular}{cccc}
\hline Fault Type & $\begin{array}{c}\text { Actual Fault } \\
\text { Distance[km] }\end{array}$ & $\begin{array}{c}\text { Fault } \\
\text { Location[km] }\end{array}$ & Error[\%] \\
\hline \multirow{4}{*}{ I AG } & I 1 & 25.74 & 4.26 \\
& I 2 & 30.13 & 0.13 \\
& 01 & 30.16 & 0.16 \\
I BCG & 02 & 29.88 & 0.12 \\
& I 1 & 25.87 & 4.13 \\
& I 2 & 30.16 & 0.16 \\
I B II CG & 01 & 30.17 & 0.17 \\
& 02 & 29.89 & 0.11 \\
& I 1 & 24.76 & 5.24 \\
& I 2 & 30.18 & 0.18 \\
I A II BCG & 01 & 29.95 & 0.05 \\
& 02 & 30.23 & 0.23 \\
& I 1 & 25.11 & 4.99 \\
& I 2 & 29.89 & 0.11 \\
& 01 & 29.86 & 0.14 \\
\hline
\end{tabular}

\section{Summary}

In this paper, the characteristics of double circuit lines with asymmetrical parameters are analyzed. Two lines are divided into positive ,negative and zero sequence component by using the symmetrical component method. The positive negative sequence components are independent of each other, but there are mutual inductance between zero sequence component. The mutual inductance between the zero sequence can be eliminated by the new decoupling method and the zero sequence is divided into 
the same direction component and the reverse direction component. The new six sequence components are mutually independent. The fault location formula can be obtained based on that the sequence voltage of fault point calculated by both ends are equivalent.

Fault location method proposed in this paper not only has the advantages of the existing two terminal fault location method, but also not be effected by fault location, fault type and transition resistance. It owns high precision and can be applied to fault location of partly coupling double circuit lines and multiple circuit lines, which has important practical significance.

\section{References}

[1] Shu Hongchun, Liu Zhensong, Peng Shixin. Single terminal fault location for two parallel transmission lines on same tower [J]. Electric Power Automation Equipment,2008,28(12):11-15.

[2] Li Xiaopeng, He Zhengyou, Xia Lulu. Natural Frequencies based fault location algorithm for parallel transmission lines on the same tower $[\mathrm{J}]$. Automation of Electric Power System, 2011,35(12):47-51.

[3] Liu Qiankuan, Li Yongbin, Huang Shaofeng. Single line fault location of parallel double transmission line on same tower based on two-terminal electrical quantities [J]. Power System Technology,2008,32(3):27-30.

[4] Yang Bo, Wei Zhinong, Wang Huafang, et al. Two terminal fault locating algorithm for double-circuit lines on the same tower based on distributed parameter model using asymmetrical parameter [J]. ELAY,2005,33(5):8-11.

[5] Wang Anning, Chen Qing, Zhou Zhanping, Tang Shaowei. An improved phase component method for calculating faults on the double-circuit transmission lines on the same tower [J]. Automation of Electric Power System,2009,33(13):58-62+77.

[6] Gu Yan, Wang Yuzhong. Fault location based on wavelet transform and mathematical morphology for double-circuit transmission lines [J]. Electric Power Automation Equipment, 2011,31(12):34-38.

[7] Zhang Yanxia, Wang Yan, Wu Shi, et al. Phase-selection for six-phase power transmission system based on six-sequence components method [J]. Automation of Electric Power System, 2009,33(24):49-53.

[8] Liu Ling, Fan Chunju. Fault calculation for joint four transmission lines of different voltage grades on the same tower based on six-sequence component method [J]. Power System Protection and Control,2010,38(9);6-11.

[9]Fan Chunju, Cai Huarong, Yu Weiyong. Accurate fault location for parallel transmission line based on six-sequence fault components [J]. Journal of Shanghai Jiaotong University, 2004, 38(8): 1279-1282.

[10]Xu Kan, Fan Chunju, XuJinxi. Study on calculation methods of ground fault through transition resistance [J]. Power System Protection and Control,2014,42(7):44-51.

[11]Xia Yi, Tai Nengling, Fan Chunju, Yu Zhongan. Four zero-sequence component method of asymmetry-parameter four-parallel lines on the same tower and its fault analysis [J]. Power System Protection and Control,2013,41(18):9-16. 